\title{
Media to Improve the Performance of Fibrous Filters ${ }^{\dagger}$
}

\author{
Anna Jackiewicz ${ }^{1 *}$, Albert Podgórskii ${ }^{1}$, Leon Gradon ${ }^{1}$ and Jakub Michalski ${ }^{2}$ \\ ${ }^{1}$ Faculty of Chemical and Process Engineering, Warsaw University of Technology, Warsaw, Poland \\ ${ }^{2}$ Faculty of Materials Science, Warsaw University of Technology, Warsaw, Poland
}

\begin{abstract}
The main objective of this study was to present one of the possibilities of increasing the initial efficiency of aerosol particle removal from the gas stream, i.e. the application of nanofibers in the filter structure. The modified melt-blown technique of producing such thin fibers is hereby presented. During this work, various filter media with fiber diameters from micro- to nanosized made of selected polymers differing in melt flow index were obtained via this method. Their morphology was precisely analysed using high-resolution equipment. The crucial parameters characterizing those fibrous materials were determined: an initial pressure drop across the filters and an initial filtration efficiency. The behavior of clean filters composed of microfibers were compared with the behavior of those made of nanofibers. An attempt was made to decrease the diameter of fibers to the nano range by using different materials for their formation. Collected data of penetration for melt-blown fibrous media were described via experimentally verified realistic models for better simulation of the air filter's performance. Additionally, the obtained results were compared with predictions of the classic filtration theory predominating in the literature on the subject.
\end{abstract}

Keywords: fibrous filter, filtration efficiency, melt-blown, nanofibers

\section{Introduction}

In the current state of environmental pollution, especially air and water, effective filtration is becoming increasingly significant. The high-quality filtration materials are also needed in industries applying so-called clean technologies. The requirements for the development and evaluation of such media are becoming more and more stringent. Among all the devices intended to separate small particles from gas streams, fibrous filters are the most widely used. Economically they are the most interesting, since they are both effective and quite simple to operate. By employing fibrous filters, one can remove even very fine particulates from gas with a high efficiency and at a relatively low pressure drop. There is a lot of evidence in the literature showing that fibrous filters can

Accepted: July 9, 2012

1,2 Waryńskiego 1 Street, 00-645 Warsaw, Poland

* Corresponding author:

E-mail: jackiewicz@ichip.pw.edu.pl

TEL: +48-22-234-62-47 successfully remove nanoparticles from the gas $^{1,2,3)}$, and they therefore penetrate new markets, expanding into new branches of industry. Performance of these filtering media, defined through their filtration efficiency, pressure drop and lifetime, strongly depends on their structure - as can be seen from the equation derived on the basis of classic filtration theory. This formula expresses the overall collection efficiency of the filter as follows: $\eta=1-\exp \left[(-4 \alpha E L) /\left(\pi d_{F}(1-\right.\right.$ $\alpha))]$. Hence one can see that the fiber diameter and the filter porosity determine the quality of the filter.

To produce an optimal filter structure for a given application, it is necessary to have extensive knowledge of the filter's formation, the clean filter's characteristics and of its behavior during loading. Our work was focused on the basic issue, i.e. an improvement of the initial state of the filtration process by increasing the efficiency of aerosol particle removal from the gas stream, at the same time maintaining a relatively low pressure drop. There are a few methods for enhancing a fibrous filter's performance, i.e. by pleating the filter surface to increase the filter- 
ing aree and to reduce the pressure drop across the filter ${ }^{4,5)}$; by charging fibers (electret filters) - which W provides an additional mechanism of aerosol particle capture $^{6)}$, and by applying nanosized fibers which are very good particle attractors ${ }^{7,8)}$. In this paper the latter method has been analysed. The interest in nanostructured media has increased rapidly in the last decade. The specialty filtration applications of nanofibrous media are featured in the work by Barhate and Ramakrishna ${ }^{9}$. Many of them have already been commercialized and some are still in development. The potential of nanofibers in filtration manifested by a high filtration efficiency and dust-holding capacity is the subject of many publications ${ }^{10,11)}$. Podgórski et al. ${ }^{12)}$ confirmed, both theoretically and experimentally, that the most penetrating particle size (MPPS) can be significantly reduced with the use of nanofibrous media. Chronakis ${ }^{13)}$ presented a review about the active research area of producing ceramic and composite nanofibers with various compositions and properties. Recent trends that can be observed in the works concerning filtration with the use of nanofibers are their surface modification ${ }^{14,15)}$ and the study of multilayer systems with a nanolayer ${ }^{16,17)}$. Graham et al. ${ }^{18)}$ discussed a process for making nanofibers, as well as the benefits, limitations, construction, and performance of filters composed of nano-sized fibers. There are two main techniques for the formation of such thin fibers, i.e. electrospinning and melt-blown. Although electrospinning makes it possible to obtain very thin fibers, it has significant drawbacks such as formation of a huge amount of toxic solvent vapor and a very low production rate. These disadvantages are eliminated in the very stable and controllable advanced melt-blown method developed by Gradon ${ }^{19,20)}$, which we use to manufacture our filters. Utilizing this most promising technique and through selection of the appropriate process conditions, the filters of desired structure - for a certain application - i.e. with defined average fiber diameter and desired mean porosity can be obtained. The influence of the process parameters on the properties of the final product can be found in the works by Bresee ${ }^{21)}$, Gradon et al. ${ }^{20)}$ and Ellison et al. ${ }^{22)}$. Tan et al. ${ }^{23)}$ studied an impact of melted polymer viscosity and elasticity on the diameter distribution of melt-blown filters.

Within the framework of this study we tried to use selected polymers differing in melt flow index to produce filaments with increasingly smaller sizes. The structure of the obtained filters, primarily the polydisperse fiber size distribution which is typical for the melt-blown filtering media, was precisely analysed using advanced techniques available in the university' s laboratories. This procedure plays a significant role in the investigations of filters, because the nature of the fiber size distribution provides valuable clues about the mechanism of the filtration process. Then, the crucial parameters characterizing tested media were determined: an initial pressure drop across the filters and an initial filtration efficiency. An attempt was made to describe the obtained data. There would be no difficulty if the filters had a homogeneous structure - i.e. are made of identical fibers uniformly distributed in a filter space - which assumes the classic approach. However, melt-blown fibrous filters are non-uniform and this fact strongly affects their performance. Therefore, a new mathematical model was used to approximate the experimental values of penetration through the polydisperse filters. The obtained results were compared with predictions of the classic filtration theory predominating in the literature on the subject.

\section{Experimental Protocol}

In this section, the method of producing fibrous filtering media as well as the experimental procedure used to characterize their structure and to study their performance are described. All tests performed within this work were conducted in accordance with the "art of measuring", taking statistics into account.

\subsection{Production method for fibrous filters}

We developed a modified melt-blown technology which allows us to produce filters composed of fibers with various diameters from micrometer to nanometer in size. The controlled formation of fibers of assumed diameter of the nanosized range is possible due to processing the raw polymer in the extruder, including the reaction with dopants which changes the lengths of the original polymer chains. It results in the reduction of the viscosity of a melted polymer introduced into the fibrillization die.

The melt-blown technology uses only the polymer as the object of the processing. The rate of fiber production with this method is much higher than for the electrospinning process. The melt-blown technique also has an additional advantage over the electrospinning in which a significant amount of the solvent vapor is produced. It requires application of a separation system for solvent recovery. The schematic of the test stand for obtaining filaments by blowing a melted polymer is shown in Fig. 1, while some details of the die construction are shown in Fig. 2(a). 


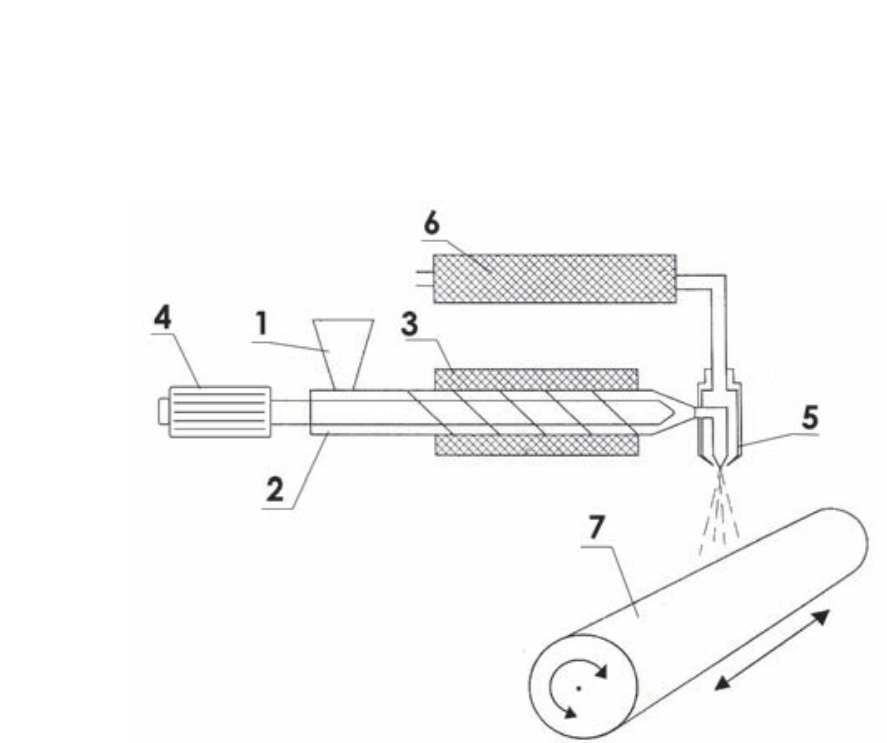

Fig. 1 System for production of melt-blown fibrous materials.
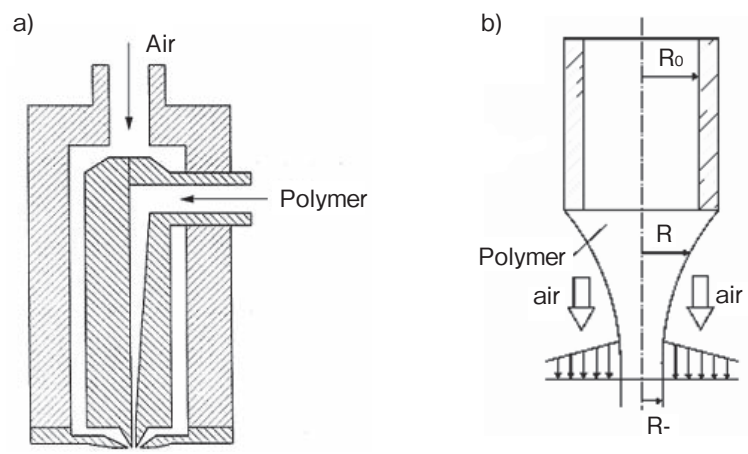

Fig. 2 Diagram showing the die structure a) and single fiber formation $b$ ).

Granulated polymer placed in a container 1 falls into the screw of the extruder 2 and is pressed into the die 5. During transportation, the polymer is heated from outside in three steps by an electronic heater 3. The flow rate of melted and homogenized polymer is determined by the rate of screw rotation controlled via an inverter by means of an electronic motor with the gear system 4 . The power transmission system is protected from the heating zone by the cooling system. The melted polymer is extruded through the row of the die nozzles. Pressure and temperature of the polymer in the die are measured with the sensing elements. In the die structure, the polymer nozzles are surrounded by the air nozzles. The stream of hot air from compressor and heater 6 flowing along the melted polymer filaments extends them to the desired diameter. The solidified fibers are collected on the mandrel of the fiber receiver 7 . This mandrel rotates and moves to and fro to form a proper filter structure defined by the fiber diameter and the local packing density of fibers.
It would be desirable to model the process of single-fiber formation in the melt-blown technology for a better understanding of its principles and for optimization of the operating conditions of fiber manufacture to ultimately obtain the expected filter structure. Let us consider a single hole of the die, Fig. 2 (b), and polymer flowing at volumetric flow rate $Q$ through it. After leaving the hole, a melted fiber of radius $R$ is formed. The basic information describing formation of a thin fiber in the melt-blown technology is derived under the following assumptions: a fiber has a cylindrical shape, a polymer has the same density for melted and solid phases, an axial velocity within a melted fiber is uniform in the fiber crosssection, the polymer is a Newtonian liquid with an Arrhenius type of dependence of viscosity on temperature, the heat capacity of the polymer is constant, the temperature distribution is uniform in the fiber cross-section, the heat conduction in axial direction is negligible.

Taking into account the constancy of the polymer volumetric flow rate, balancing the momentum, and using the stress-strain relations and energy balances, we were able to estimate the influence of the process parameters on the properties of the final product. Theoretical analysis of the single-fiber formation gives us the quantitative information about the radius $R$ of the solidified polymer fiber at the distance $x$ from the nozzle hole of radius $R_{o}$ for precisely fixed model parameters, i.e. polymer constitutive properties, flow rates and temperatures of polymer and air, and polymer pressure in the die. Using this information, the process parameters for the melt-blown technology were defined.

\subsection{Filter media characterization}

During this work, the desired melt-blown filter layers were produced for a fixed set of process parameters and were used in the filtration testing procedure. At the beginning, their structure was analysed, because the more we know about the non-uniform nature of the filter material, the better we can predict its performance (filtration efficiency and pressure drop). The following parameters of the tested filters were determined: filter thickness, $L$, packing density, $\alpha$ , basic weight, $q_{s}$. Filter thickness was measured using the precise vernier caliper, and the values of the last two parameters were evaluated by a gravimetric method. Determining the mass of a filter sample, $m_{F}$, the surface density was calculated as the ratio of the mass to the filter area, $F: q_{s}=m_{F} / F$. The filter porosity, $\varepsilon$, was determined according to the presented 
farmulga? $\varepsilon=\left[1-\left(m_{F} / \rho_{F} \cdot F \cdot L\right)\right]^{*} 100 \%$ and then the filter solifity was computed as: $\alpha=1-\varepsilon$.

Because one of the main structural parameters of a melt-blown fibrous filter is the fiber diameter, the procedure of determining the fiber size distribution requires high accuracy and very good equipment, especially if a filter with a significant fraction of nanofibers is considered. The arithmetic mean fiber diameters, $d_{F a}$, and observed range of fiber sizes were obtained by analysing images taken under the High-Resolution Scanning Electron Microscope (HRSEM), Hitachi Su-8000, with field emission gun. The flat rectangular specimens of dimensions $10 \times 15 \mathrm{~mm}$ were cut by laser from different parts of the investigated filters. For each specimen, 16 images were acquired at magnifications varying from 300 to 2000 .

On the basis of the literature data ${ }^{22,24)}$ and structural analysis of our filters, it can be stated that in most cases, the distribution of fiber diameters of melt-blown fibrous filters follows the log-normal one that has the normalized density distribution function described as:

$$
g\left(d_{F}\right)=\frac{1}{\sqrt{2 \pi} d_{F} \ln \sigma_{g d_{F}}} \exp \left[-\frac{\ln ^{2}\left(d_{F} / d_{F g}\right)}{2 \ln ^{2} \sigma_{g d_{F}}}\right]
$$

and the normalized cumulative log-normal distribution given by:

$$
G\left(d_{F}\right)=\frac{1}{2}\left\{1+\operatorname{erf}\left[\frac{\ln \left(d_{F} / d_{F g}\right)}{\sqrt{2} \ln \sigma_{g d_{F}}}\right]\right\}
$$

This distribution is characterized by two parameters: - the geometric mean fiber diameter, $d_{F g}$ :

$$
d_{F g}=\left(d_{F 1} \times d_{F 2} \ldots \times d_{F N_{F}}\right)^{1 / N_{F}}
$$

where $N_{F}$ is the number of measured fiber diameters, - the geometric standard deviation, $\sigma_{g d F}$ :

$$
\ln \sigma_{g d_{F}}=\sqrt{\frac{\sum_{j=1}^{j=N_{F}}\left(\ln d_{F j}-\ln d_{F g}\right)^{2}}{N_{F}-1}}
$$

where $d_{F F}, j=1, \cdots, N_{F}$.

These parameters were determined by fitting the experimental data of the fiber diameters to the normalized cumulative log-normal function, Eq. 2. Even for a few measured fiber diameters, the applied procedure allows one to find the geometric mean fiber diameter and geometric standard deviation with a high accuracy.
The fact that the fiber diameter distribution of nonwoven filters can be precisely described by a log-normal distribution was utilized by us in mathematical calculations during estimation of the filter penetration (see Section 5).

In the next stage, media were examined with a Palas MFP 2000 test bench to determine the initial pressure drop across the filters and their initial filtration efficiency. Arizona Fine Test Dust ISO 12103-1 (Power Technology Incorporated, USA) was used as the standard test particles in the size range of submicrometers and micrometers. The experiments were performed at an air velocity of $0.2 \mathrm{~m} / \mathrm{s}$.

\section{Impact of the Filter Morphology on the Filtra- tion Effectiveness}

The first task was related to comparison of the experimental data of filtration properties obtained for two filters with different morphology. Their structural parameters are shown in Table 1. Fig. 3 depicts the fiber size distributions of the tested filters together with their precise approximation by the log-normal distribution.

The presented in Fig. 4 results show the influence of the fiber diameter distribution and porosity of the filter on its effectiveness. It was tested whether the application of thinner fibers in the filter can increase its efficiency and whether simultaneously one has to pay for this with an excessive pressure drop. On the basis of the obtained data it can be seen that Filter 1 with thinner fibers, low basic weight and less packed provides nearly three times higher efficiency than Filter 2 with thicker fibers, higher basic weight and lower porosity. This difference is particularly visible for the most penetrating particles. The increase in pressure drop for the filter with thin filaments in comparison to the pressure drop for the filter with thicker fibers is not so significant. This is the evidence that thinner fibers in a filter have a beneficial effect on the separation efficiency of aerosol particles but they also cause an increase in pressure drop. Therefore, when optimizing the filter structure, two main parameters characterizing the filters, i.e. the filtration efficiency and pressure drop across the filter, must be taken into account.

Table 1 Structural characteristic of the investigated filters

\begin{tabular}{ccccc}
\hline Filter No. & $\begin{array}{c}\text { Filter } \\
\text { thickness, } \\
L[\mathrm{~mm}]\end{array}$ & $\begin{array}{c}\text { Filter } \\
\text { porosity, } \\
\varepsilon[\%]\end{array}$ & $\begin{array}{c}\text { Basic weight, } \\
q_{\mathrm{s}}\left[\mathrm{g} / \mathrm{m}^{2}\right]\end{array}$ & $\begin{array}{c}\text { Arithmetic mean } \\
\text { fiber diameter, } \\
d_{F a} \pm \sigma_{a d f}[\mu \mathrm{m}]\end{array}$ \\
\hline 1 & 2.05 & 97.5 & 39 & $3.5 \pm 1.7$ \\
\hline 2 & 1.35 & 85.0 & 187 & $15.4 \pm 6.7$ \\
\hline
\end{tabular}



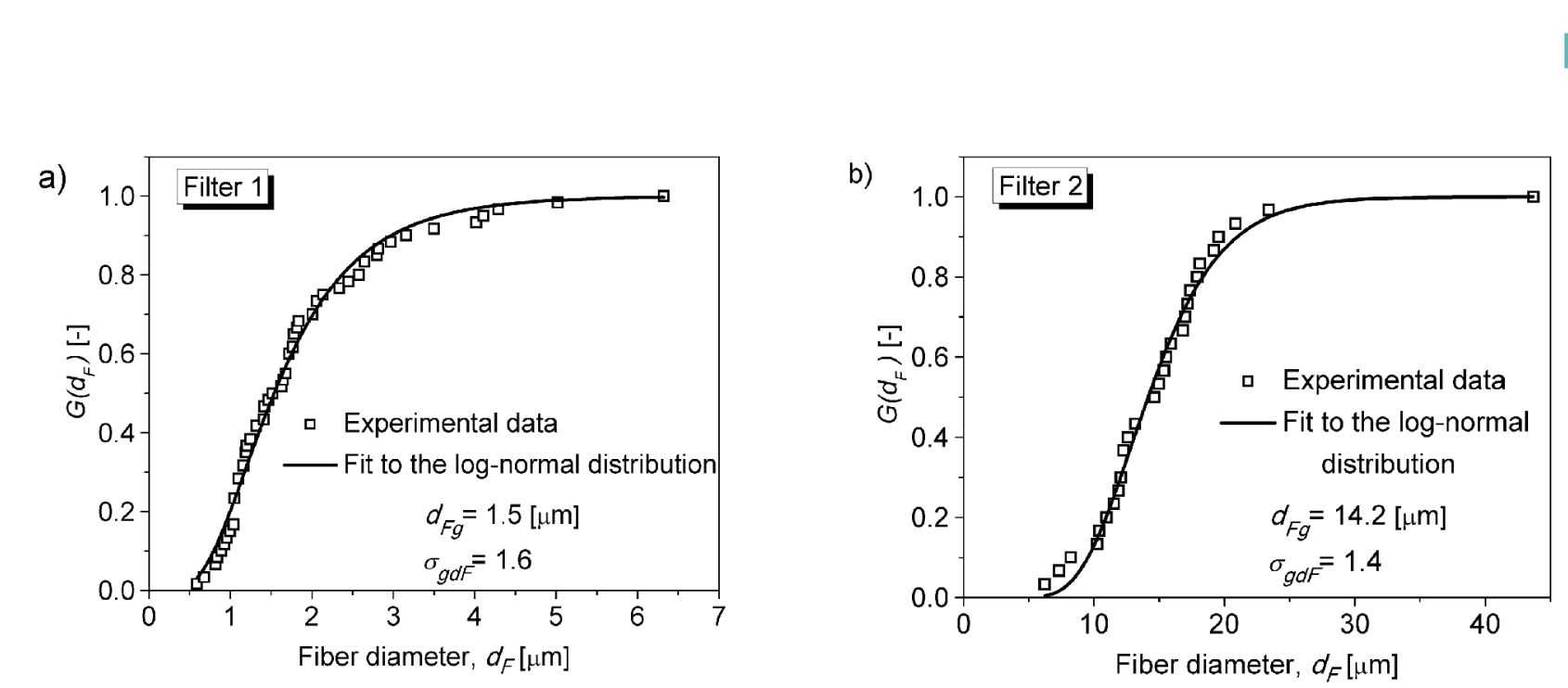

Fig. 3 Fiber size distributions of Filter 1 (a) and Filter 2 (b).

a)

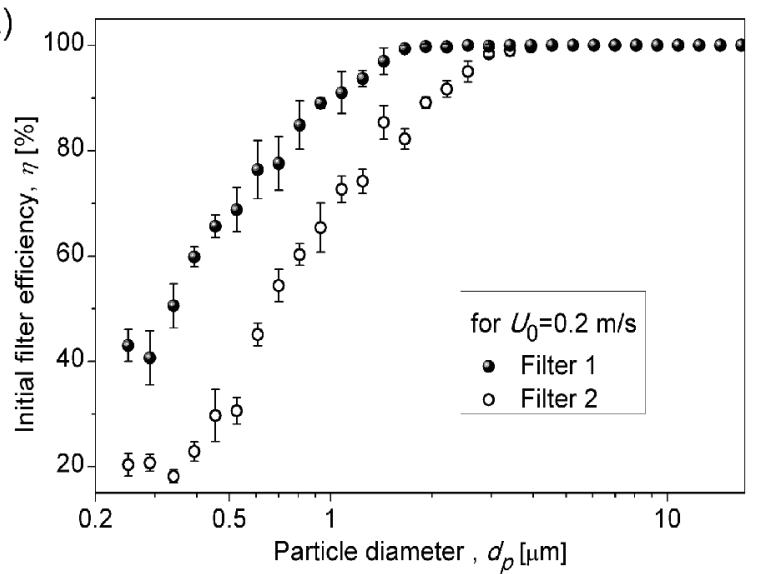

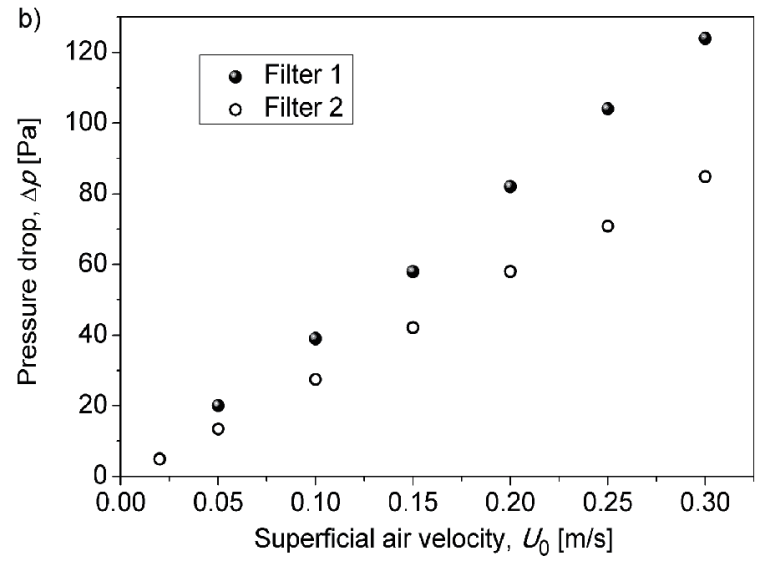

Fig. 4 Initial filter performance: a) filter efficiency vs. particle diameter and b) pressure drop vs. face velocity for Filter 1 and Filter 2.

\section{Impact of Material Used to Manufacture Fil- ters for Fiber Diameters}

It was proven that the thin fibers in the filter ensure their better quality. A further step in the study was to attempt to reduce the fiber diameter and produce filters with a substantial fraction of nanosized fibers. The investigations of the melt-blown technique for fibrous media production were extended to the case of the polymers with different viscosity, i.e. different values of the melt flow index (MFI). Two kinds of polypropylene were used for the fiber formation, namely Borealis PP504FB with MFI 1200, and Metocene PPM650Y with MFI 1800. The process was carried out using the same structure of the experimental equipment, i.e. the screw of the extruder and construction of the die, only the pressure of the polymer in the die was changed. The filtering media obtained were thoroughly analysed in order to verify the range of fiber diameters formed from the mentioned poly-
Table 2 Characteristic of the filters made of two different polymers

\begin{tabular}{ccccc}
\hline Filter & $\begin{array}{c}\text { Filter } \\
\text { thickness, } \\
L[\mathrm{~mm}]\end{array}$ & $\begin{array}{c}\text { Packing } \\
\text { density, } \\
\alpha[-]\end{array}$ & $\begin{array}{c}\text { Basis } \\
\text { weight, } \\
q_{s}\left[\mathrm{~g} / \mathrm{m}^{2}\right]\end{array}$ & $\begin{array}{c}\text { Arithmetic } \\
\text { mean fiber } \\
\text { diameter, } \\
d_{a F} \pm \sigma_{a d F} \\
{[\mu \mathrm{m}]}\end{array}$ \\
\hline PP504FB & 3.15 & 0.016 & 34.5 & $0.70 \pm 0.68$ \\
\hline PPM650Y & 3.42 & 0.014 & 43.3 & $0.55 \pm 0.54$ \\
\hline
\end{tabular}

mers (see Table 2).

The fiber size distributions presented in Fig. 5 show the significant influence of the polymer structure, i.e. the polymer chain characteristics, defined through the melt flow index, on the diameters of produced fibers. Metocene PPM650Y with MFI 1800 makes it possible to obtain fibers with the smaller diameters. Therefore, for the lower viscosity of the polymer used to manufacture filters (the greater its $\mathrm{MFI}$ ), the thinner filaments were made.

In Fig. 6 the operating parameters at the initial stage of filtration process, determined for those two 

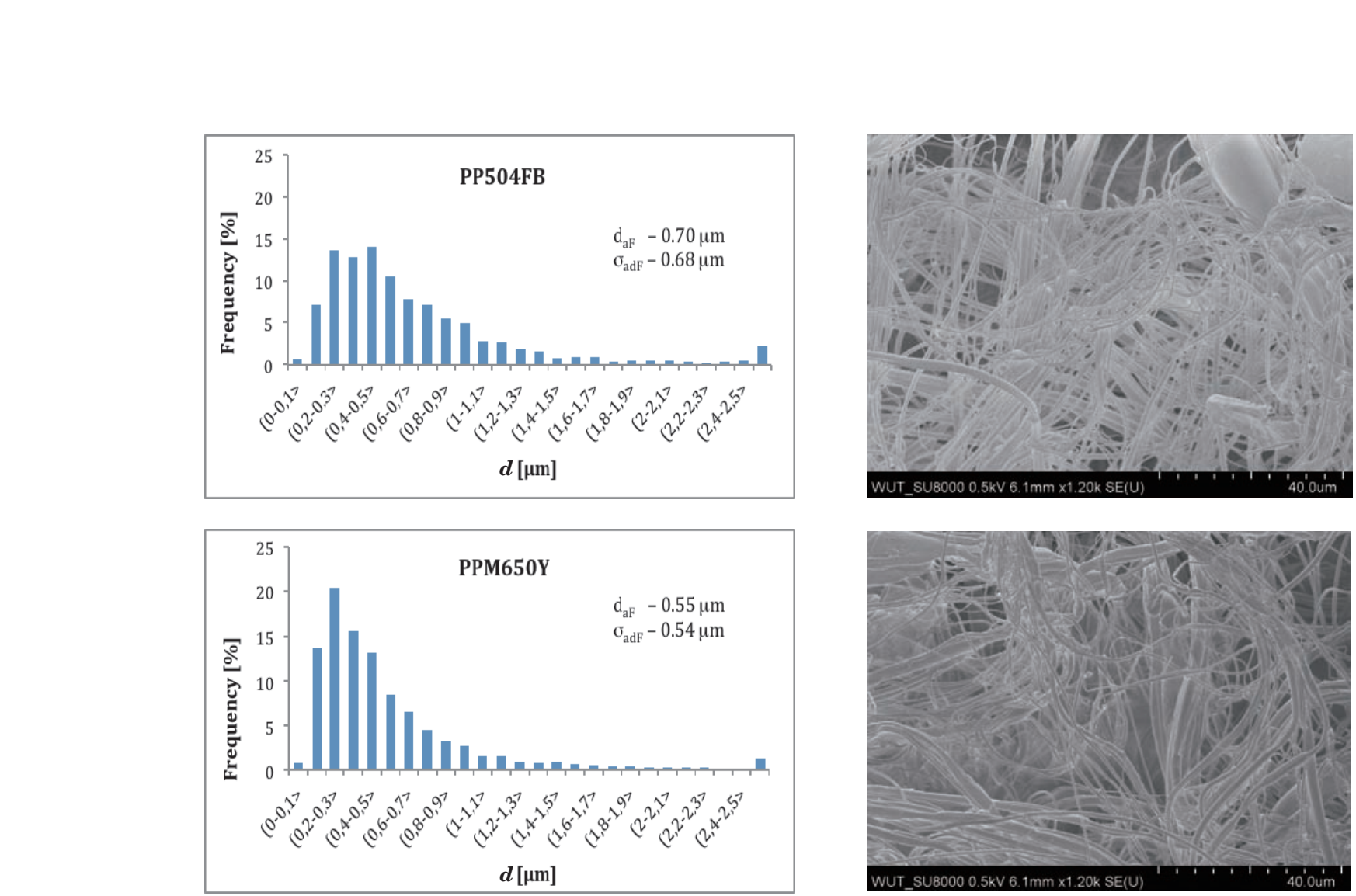

Fig. 5 HRSEM images and fiber diameter distributions of filters made of different polypropylenes: Borealis (Filter PP504FB) and Metocene (Filter PPM650Y).
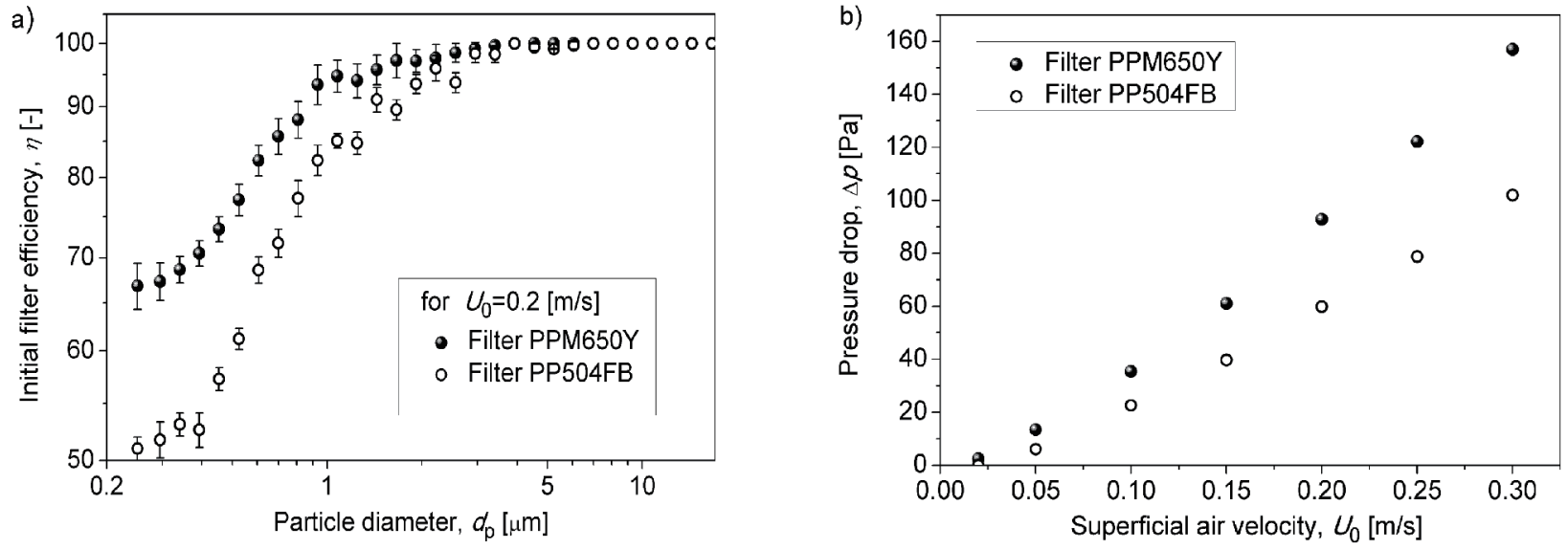

Fig. 6 Initial performance of filters made of various materials : a) filter efficiency and b) pressure drop.

filtering materials made of various polymers, were collated. It turned out that the highest contribution of nanosized fibers in the filter made of Metocene enhanced its effectiveness in comparison with the filter composed of slightly thicker fibers made of Borealis. Filter PPM650Y made of thin fibers displays a greater air-flow resistance, however, the increase compared with Filter PM504FB composed of fibers with larger diameters is not as significant.

\section{Theoretical Approach}

Aerosol filtration in fibrous filters is a very complex process. A particle's motion during flow is influenced by several stochastic and deterministic forces which act simultaneously, but depending on the particle size and process conditions, one of them may predominate. Moreover, the polydisperse internal structure of these filters creates non-uniform fields of local gas velocity and local particle concentrations in a 
givengfiten eross-section, and this is one of the major pooblems in the theoretical modeling of filtration processes using them. This intricate internal structure, extremely hard to imitate in computer simulations, causes a process of aerosol filtration in fibrous filters that is difficult to describe and which out of necessity must be based on several simplifications. In the literature on the subject, the classic approach used to predict fibrous media performance still prevails. This is without doubt due to its simplicity. It considers filtration at its most elementary level - the collection of an aerosol particle by a single fiber, placed with its axis perpendicular to the air flow. The main assumptions of the classic theory of depth filtration in fibrous filters are: macroscopic homogeneity of the filter; the same efficiency of all fibers; mutual independence of the particular mechanisms of deposition; irreversible removal of a particle upon a contact with a fiber (meaning no rebound of a particle). According to this model, an aerosol particle's penetration, $P$, through a filter with thickness $\mathrm{L}$ and packing density $\alpha$ is expressed as:

$$
P=\exp \left[-\frac{4 \alpha L}{\pi(1-\alpha)}\left(\sum_{m} \frac{E_{m}\left(d_{F}\right)}{d_{F}}\right)\right]
$$

where $E_{m}\left(d_{F}\right)$ is the single fiber efficiency due to $\mathrm{m}^{\text {th }}$ mechanism of deposition calculated additively (i.e., $E_{D}$ diffusion, $E_{R}$ interception, $E_{I}$ inertial impaction, $E_{D R}$ coupling of diffusion and interception), which depends on the fiber diameter, $d_{F}$. Equation (5) originates from the balance of the particle's flux deposited in the filter layer with a differential thickness, and such a derivation is included in works by Dorman ${ }^{25)}$, Brown $^{26)}$. The single fiber efficiencies for particular mechanisms can be determined using the correlations that may be found in the literature. Since in this work, particles with diameters in the range of 0.25 $17 \mu \mathrm{m}$ were considered, analysis was limited to the three predominating mechanisms of deposition, i.e. Brownian diffusion, direct interception and inertial impaction. In the absence of an electric charge on fibers and particles, the electrostatic mechanism was omitted. The calculations were performed using the correlations presented below.

$$
\text { For diffusion : } E_{D}=2.9\left(\frac{1-\alpha}{K u}\right)^{1 / 3} P e^{-2 / 3}
$$

where $K u=-0.5 \ln \alpha-0.75-0.25 \alpha^{2}+\alpha$ denotes the Kuwabara number, $P e=U_{0} d_{F} / D$ is the Peclet number, $D$ - the Brownian diffusion coefficient of an aerosol particle, and $U_{0}$ - the superficial mean gas velocity.
The genesis of creation of Equation (6) is contained in the work by Podgórski et al. ${ }^{27)}$.

$$
\text { For inertial impaction }^{28)}: \quad E_{I}=\frac{(S t k) J}{2 K u^{2}}
$$

when $N R<0.4 \rightarrow J=\left(29.6-28 \alpha^{0.62}\right) N R^{2}-27.5 N R^{2.8}$, when $N R \geq 0.4 \rightarrow J=2$,

where $S t k=\rho_{P} d^{2}{ }_{P} C_{C} U_{0} / 18 \mu d_{F}$ is the Stokes number, $C_{C}$ - the Cunningham slip correction factor, $\rho_{P}-$ the particle density, $\mu$ - the air viscosity, and $N R=d_{P} / d_{F}$ signifies the interception parameter.

For direct interception ${ }^{29)}$ :

$E_{R}=\left(\frac{1-\alpha}{K u}\right)\left(\frac{N R^{2}}{1+N R}\right)$

Enhancement of the single fiber efficiency caused by a coupling between diffusion and direct interception, $E_{D R}$, was also accounted for ${ }^{30)}$ :

$$
E_{D R}=\frac{1.24 N R^{2 / 3}}{(K u P e)^{1 / 2}}
$$

Use of the above-cited formula to calculate the aerosol particle penetration does not give rise to any difficulties for the filter composed of identical fibers uniformly distributed in a filter space. Nevertheless, the real nonwoven filters tend to have relatively wide distributions of fiber diameters, and application of the classic theory outlined above is not explicit. Most authors adapt this model of depth filtration derived for monodisperse media to describe the behavior of polydisperse filters by using a certain equivalent fiber diameter in calculations, e.g. the arithmetic mean diameter determined by image analysis or the equivalent diameter obtained from the pressure drop measurements. Two different filtering media previously characterized were utilized to verify applicability of this common approach for the arithmetic mean fiber diameter.

It can be observed in Fig. 7 that application of the arithmetic mean fiber diameter failed to precisely describe penetration through polydisperse meltblown filters composed of both micro- and nanosized fibers. The discrepancies between the experimental data and the predictions of the classic approach were caused by the fact that theoretical calculations had been based on the mean fiber diameter only, whilst the real filters always have a more or less polydisperse distribution of fiber sizes.

In the light of the presented results, it is obvious that in the case of inhomogeneous melt-blown fibrous filters, the classic filtration theory assuming a regular 

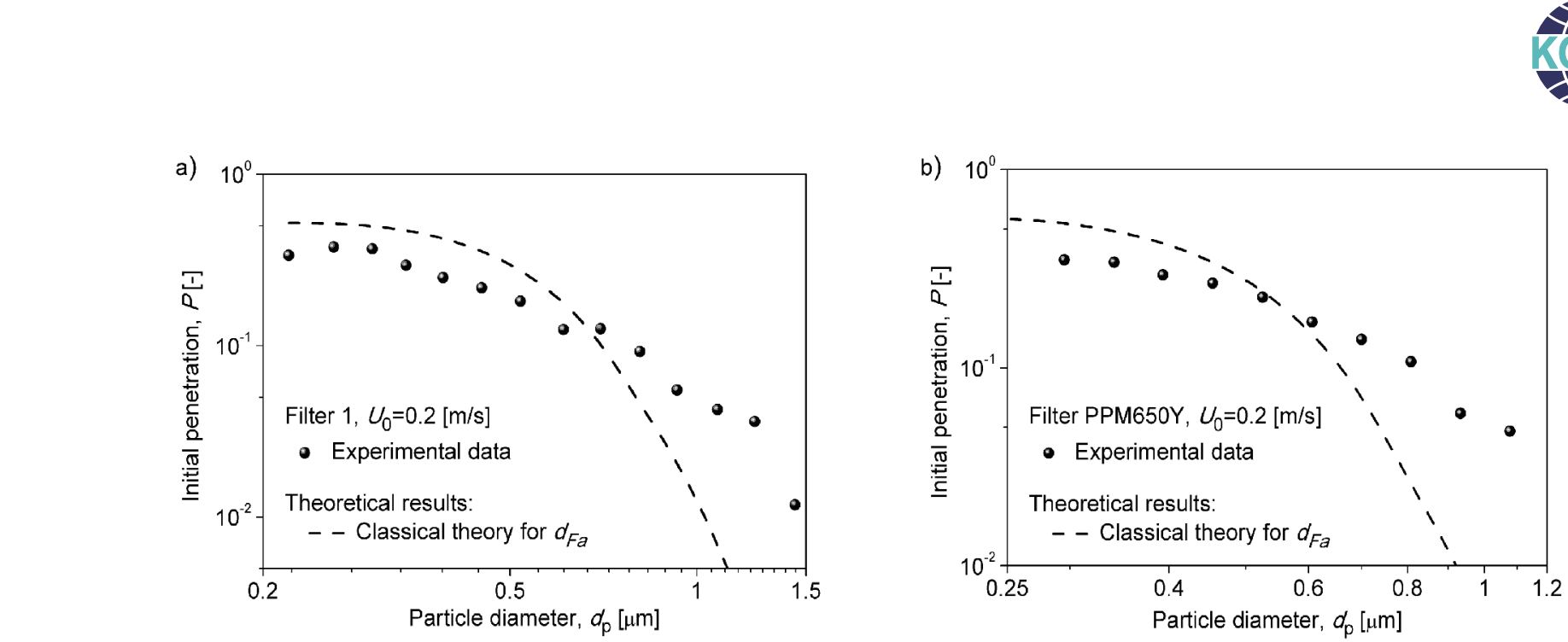

Fig. 7 Comparison of experimental values of the efficiency with results of calculations obtained using the classic theory for: Filter 1 (a) and Filter PPM650Y (b).

array of identical fibers in a filter is an oversimplification. Instead of one mean fiber diameter, the entire fiber diameter distribution should be taken into account, because aerosol penetration through a polydisperse filter depends on it. Therefore, to realistically calculate penetration of aerosol particles through polydisperse filters, theoretically well-founded models were utilized - two limiting models, namely Perfectly Mixed Flow Model (PMFM) and Fully Segregated Flow Model (FSFM), and the general model called Partially Segregated Flow Model (PSFM), which is their linear combination. The proposed models explicitly take one of the main aspects of the filter structure inhomogeneity into consideration, i.e. the entire fiber size distribution. These two limiting models estimate an upper and a lower possible aerosol penetration through a polydisperse filter, while PSFM enables determination of the real penetration, which lies somewhere in between Perfectly Mixed Flow Model and Fully Segregated Flow Model. When fibers with different sizes are distributed evenly in a filter space and flow in a filter is well mixed on the mesoscale, Eq. (5) can be simply averaged using the fiber diameter distribution function $g\left(d_{F}\right)$, Eq. (1). This method represents estimation of the lower limit of aerosol penetration through a polydisperse filter and it is called Perfectly Mixed Flow Model (PMFM). Such an approach is based on the assumption that the superficial gas velocity is the same for all $\mathrm{Ku}$ wabara cells containing various fibers. Thus, aerosol penetration according to $P M F M$ is calculated as:

$$
P_{P M F M}=\exp \left[\frac{-4 \alpha L}{\pi(1-\alpha)}\left(\sum_{m} \int_{0}^{\infty} \frac{E_{m}\left(d_{F}\right)}{d_{F}} g\left(d_{F}\right) \mathrm{d} d_{F}\right)\right]
$$

The second extreme model estimating the upper limit of aerosol penetration through a polydisperse filter considers the situation when fibers with different diameters are completely separated from each other. Assuming that the pressure drop per unit filter thickness is the same for all Kuwabara cells with various fibers, the Fully Segregated Flow Model $(F S F M)^{31)}$ was formulated and the penetration according to this model is expressed as:

$$
P_{F S F M}=\frac{\int_{0}^{\infty} d_{F}^{2} P\left(d_{F}\right) g\left(d_{F}\right) \mathrm{d} d_{F}}{\int_{0}^{\infty} d_{F}^{2} g\left(d_{F}\right) \mathrm{d} d_{F}}
$$

$P\left(d_{F}\right)$ in the above formula denotes penetration calculated using Eq. (5) as for a monodisperse filter with fibers of diameter $d_{F}$, and the single fiber deposition efficiencies $E_{m}\left(d_{F}\right)$ for particular fibers' diameters should be computed for individual superficial gas velocities $U_{0}\left(d_{F}\right)$, corresponding to various Kuwabara cells with different fibers:

$$
U_{0}\left(d_{F}\right)=\frac{U_{0} d_{F}^{2}}{\int_{0}^{\infty} d_{F}^{2} g\left(d_{F}\right) \mathrm{d} d_{F}}
$$

The actual value of penetration for a specific filter for a given degree of mixing of fibers with different diameters lies between $P_{P M F M}$ and $P_{F S F M}$ and depends on the degree of segregation of the aerosol flow in a filter around the fibers of different sizes. Thus, we formulated the general Partially Segregated Flow Model $(P S F M)$, for which the penetration is calculated as a linear combination of penetrations determined from the two limiting models - Eq. (10) and (11):

$$
P_{P S F M}=s P_{F S F M}+(1-\mathrm{s}) P_{P M F M}
$$



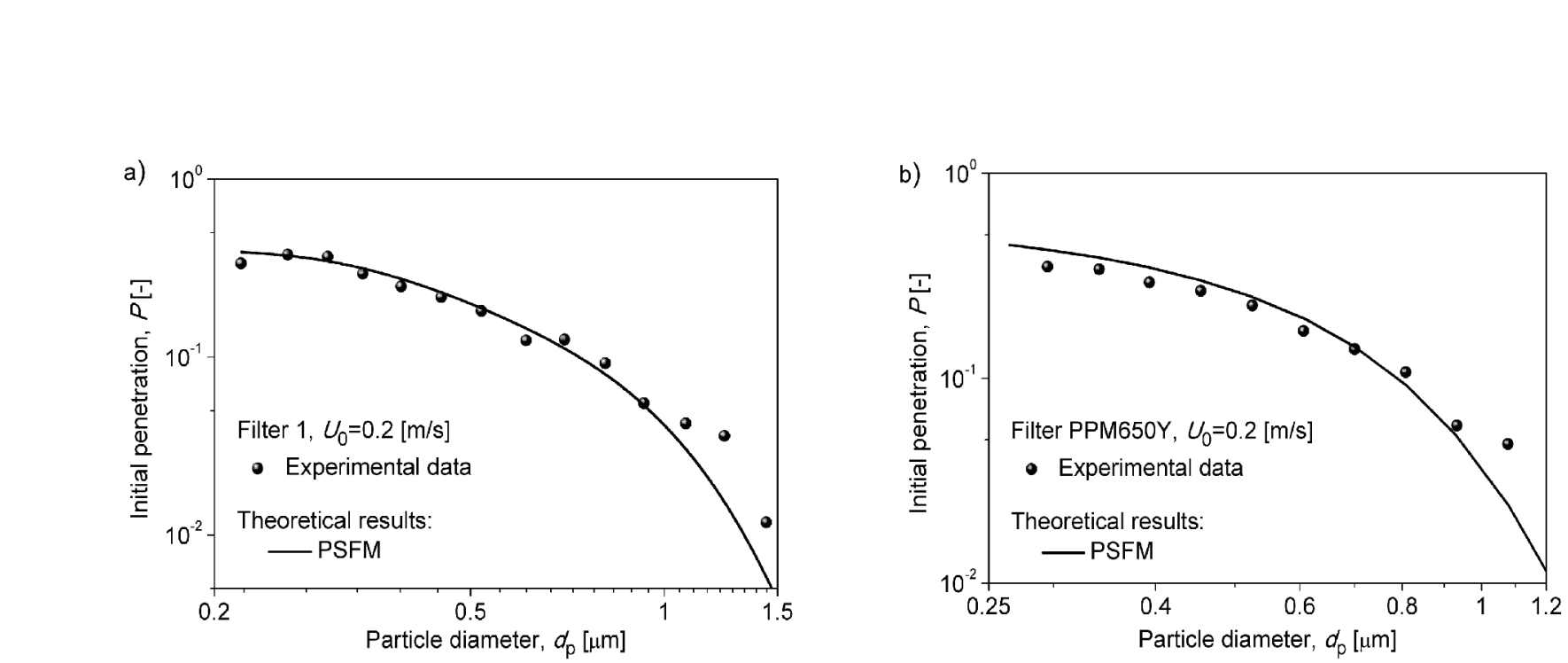

Fig. 8 Partially Segregated Flow Model predictions vs. experimental data of penetration for: Filter 1 (a) and Filter PPM650Y (b).

In Eq. (13) the dimensionless, phenomenological parameter named the flow segregation intensity, s, was introduced. It can vary between zero (for perfect mixing) and one (for full segregation) and must be determined on the basis of experimental penetration data.

The experimental results of submicrometer and micrometer aerosol particle penetration through the same two filters whose data were compared with results of the classic theory, were described by the Partially Segregated Flow Model, see Fig. 8. As it can be observed, utilization of the entire fiber size distribution in theoretical calculations, i.e. using the $P S F M$, enables the particle penetration through filters made of both micro- and nanometer sized fibers to be computed much more precisely compared to the common case when only a mean fiber diameter is considered. The latter approach leads to results which diverge tremendously from experimental data and can be either lower or higher than them. The obtained results clearly indicate how strong the effect of the fibers' polydispersity on the filtration of aerosol particles with various sizes can be. Podgórski et al. ${ }^{27}$ also successfully applied this model for prediction of the nanoparticles' penetration.

\section{Conclusions}

In this paper, the way to enhance the dust removal efficiency of fibrous filters by applying nanostructured fibers was presented. We introduced various modifications of the classic melt-blown technique in order to produce fibers with a wide range of sizes. The production parameters have been organized in order to obtain a filter with an assumed structure. Our efforts were particularly directed towards the preparation of media with a high contribution of nanofibers and to identify the role they play during filtration. The analysis of the obtained filter structure made using the highest quality equipment confirmed that we are able to significantly decrease the average fiber diameter to below $1 \mu \mathrm{m}$ by modulating several processing parameters and by using various materials. It was shown that by designing a filter with nanofibers, one can greatly increase the efficiency of the separation of aerosol particles compared to filters composed of thicker fibers, something which is particularly noticeable in the case of the most penetrating particle size range. An attempt to reduce the fiber diameter using a new polymer with a different melt flow index was successful. Metocene with a higher MFI gives the possibility of obtaining a filter with a higher fraction of fibers less than $1 \mu \mathrm{m}$, which improves its effectiveness. Our work also examines the theoretical aspect of filtration in fibrous materials, i.e. contains a description of the obtained penetration via two models: the classic one from the literature and the one proposed by us. The results obtained clearly demonstrate that the classic single fiber theory based on the assumption of a homogeneous filter structure and monodisperse fiber size is incapable of correctly predicting the filter efficiency for real fibrous filters with a high level of polydispersity in fiber sizes. A very good agreement between the experimental data of penetration and the theoretical PSFM model was obtained for two different filters composed of micro- and nanosized fibers. It is of great practical importance to be able to precisely calculate filtration efficiency for polydisperse filters made of fibers with various diameters. This work enables us to work out general procedures for designing and optimizing fibrous filters used for different applications. 
arfis. Work will be extended to the case of the nonstedady-state filtration where the dust capacity of W the filter will be the challenging parameter for filter structure optimization.

\section{Acknowledgements}

This work was partially supported by Cummins Filtration, USA, and by the project from The National Centre for Research and Development EraNet/ MNT/NFSM/1/2011.

\section{Nomenclature}

$C_{C} \quad$ Cunningham slip correction factor, dimensionless

$D \quad$ coefficient of Brownian diffusion, $\mathrm{m}^{2} \cdot \mathrm{s}^{-1}$

$d_{F} \quad$ fiber diameter, $\mathrm{m}$

$d_{F a} \quad$ arithmetic mean fiber diameter, $\mathrm{m}$

$d_{F g} \quad$ geometric mean fiber diameter, $\mathrm{m}$

$d_{p} \quad$ particle diameter, $\mathrm{m}$

$E$ total single fiber efficiency, dimensionless

$E_{D} \quad$ single fiber efficiency due to diffusion, dimensionless

$E_{D R} \quad$ single fiber efficiency due to diffusion and interception, dimensionless

$E_{I} \quad$ single fiber efficiency due to inertial impaction, dimensionless

$E_{m} \quad$ single fiber efficiency for $m^{\text {th }}$ mechanism of deposition, dimensionless

$E_{R} \quad$ single fiber efficiency due to direct interception, dimensionless

$F \quad$ filter area, $\mathrm{m}^{2}$

$L \quad$ filter thickness, $m$

$m_{F} \quad$ mass of a filter sample, $\mathrm{kg}$

$N_{F} \quad$ number of measured fiber diameters, dimensionless

$N R \quad$ interception parameter, dimensionless

$P$ particle penetration through a filter, dimensionless

$\Delta p \quad$ pressure drop across the filter, $\mathrm{Pa}$

$q_{s} \quad$ basic weight, $\mathrm{kg} \cdot \mathrm{m}^{-2}$

$R \quad$ radius of a fiber, $\mathrm{m}$

$R_{0} \quad$ radius of a nozzle hole, $\mathrm{m}$

S aerosol particles'segregation intensity parameter, dimensionless

$U_{0} \quad$ superficial gas velocity, $\mathrm{m} \cdot \mathrm{s}^{-1}$

Greek

$\alpha \quad$ filter packing density, dimensionless

$\varepsilon \quad$ filter porosity, dimensionless

$\eta \quad$ filter efficiency, dimensionless $\mu \quad$ gas viscosity, $\mathrm{Pa} \cdot \mathrm{S}$

$\rho_{F} \quad$ fiber material density, $\mathrm{kg} \cdot \mathrm{m}^{-3}$

$\rho_{g} \quad$ gas density, $\mathrm{kg} \cdot \mathrm{m}^{-3}$

$\rho_{p} \quad$ particle density, $\mathrm{kg} \cdot \mathrm{m}^{-3}$

$\sigma_{a d F} \quad$ standard deviation of fiber size distribution, $\mathrm{m}$

$\sigma_{g d F} \quad$ geometric standard deviation of fiber size distribution, dimensionless

Dimensionless numbers

$\begin{array}{ll}\mathrm{Ku} & \text { Kuwabara number } \\ \mathrm{Pe} & \text { Peclet number } \\ \text { Stk } & \text { Stokes number }\end{array}$

\section{References}

1) Payet, S. et al. (1992): Penetration and pressure-drop of a Hepa filter during loading with submicron liquid particles, Journal of Aerosol Science, Vol.23, pp.723735 .

2) Brown, R. C. and Thorpe, A. (2001): Glass fiber filters with bimodal fiber size distribution, Powder Technology, Vol.118, pp.3-9.

3) Huang, S. H. et al. (2007): Penetration of $4.5 \mathrm{~nm}$ to 10 $\mu \mathrm{m}$ aerosol particles through fibrous filters, Journal of Aerosol Science, Vol.38, pp.719-727.

4) Rebaia, M. et al. (2010): Clogging modeling in pleated filters for gas filtration, Chemical Engineering Research and Design, Vol.88, pp.476-486.

5) Cesar, T. and Schroth, T. (2002): The Influence of Pleat Geometry on the Pressure Drop in Deep-pleated Cassette Filters, Filtration and Separation, November 2002, pp.48-54.

6) Chazelet, S., Bemer, D. and Grippari, F. (2011): Effect of the test aerosol charge on the penetration through electret filter, Separation and Purification Technology, Vol.79, pp.352-356.

7) Wang, J., Kim, S. C. and Pui, D. Y. H. (2008): Figure of merit of composite filters with micrometer and nanometer fibers, Aerosol Science and Technology, Vol.42, pp.722-728.

8) Yun, K.M. et al. (2007): Nanoparticle filtration by electrospun polymer fibers, Chemical Engineering Science, Vol.62, pp.4751-4759.

9) Barhate, R. S. and Ramakrishna, S. (2007): Nanofibrous filtering media: Filtration problems and solutions from tiny materials, Journal of Membrane Science, Vol.296, pp.1-8.

10) Barhate, R.S. et al. (2008): Fine chemical processing: The potential of nanofibres in filtration, Filtration and Separation, Vol.45, pp.32-35.

11) Hung, C. and Leung, W. W. (2011): Filtration of nanoaerosol using nanofiber filter under low Peclet number and transitional flow regime, Separation and Purification Technology, Vol.79, pp.34-42.

12) Podgórski, A., Bałazy, A. and Gradoń, L. (2006): Appli- 
ion 9 . nanofibers to improve the filtration efficiency offore most penetrating aerosol particles in fibrous W'ilters, Chemical Engineering Science, Vol.61, pp.68046815.

13) Chronakis, I. S. (2005): Novel nanocomposites and nanoceramics based on polymer nanofibers using electrospinning process-A review, Journal of Materials Processing Technology, Vol.167, pp.283-293.

14) Karwa, A. and Tatarchuk, B. (2012): Aerosol filtration enhancement using carbon nanostructures synthesized within a sintered nickel microfibrous matrix, Separation and Purification Technology, Vol.87, pp.8494.

15) Nguyen, T., Chung, O. and Park, J. (2011): Coaxial electrospun poly (lactic acid)/chitosan (core/shell) composite nanofibers and their antibacterial activity, Carbohydrate Polymers, Vol.86, pp.1799-1806.

16) Leung, W. W., Hung, C. and Yuen, P. (2010): Effect of face velocity, nanofiber packing density and thickness on filtration performance of filters with nanofibers coated on a substrate, Separation and Purification Technology, Vol.71, pp.30-37.

17) Zhang, Q. et al. (2010): Improvement in nanofiber filtration by multiple thin layers of nanofiber mats, Journal of Aerosol Science, Vol.41, pp.230-236.

18) Graham, K. et al. (2002): "Polymeric nanofibers in air filtration applications" , Fifteenth Annual Technical Conference \& Expo of the American Filtration \& Separations Society, Galveston, Texas.

19) Ciach, T. and Gradoń, L. (1996): Highly efficient filtering materials, Journal of Aerosol Science, Vol.27, pp.613-614.

20) Gradoń, L., Podgórski, A. and Bałazy, A. (2005): Filtration of nanoparticles in the nanofibrous filters, FILTECH 2005 Conference and Exhibition, Wiesbaden, Germany. Conference Proceedings, Vol. II, pp.178-183.

21) Bresee, R. R. (2004): Influence of processing condi- tions on melt blown web structure: Part 1 - DCD, International Nonwovens Journal, Vol.13, pp.49-55.

22) Ellison, C. J. et al. (2007): Melt blown nanofibers: Fiber diameter distributions and onset of fiber breakup, Polymer, Vol.48, pp.3306-3316.

23) Tan, D. H. et al. (2010): Meltblown fibers: Influence of viscosity and elasticity on diameter distribution, Journal of Non-Newtonian Fluid Mechanics, Vol.165, pp.892-900.

24) Kirsch, A. A. and Stechkina, I. B. (1973): Pressure drop and diffusional deposition of aerosol in polydisperse model filter, Journal of Colloid and Interface Science, Vol.43, pp.10-16.

25) Dorman, R. G. (1966): "Filtration. In: Aerosol Science (C. N. Davies, Ed.)” , Academic Press, London, pp.195-222.

26) Brown, R. C. (1993): "Air Filtration: An integrated approach to the theory and applications of fibrous filters" , Pergamon Press, Oxford.

27) Podgórski, A. et al. (2011): Penetration of monodisperse, singly-charged nanoparticles through fibrous filters, Aerosol Science and Technology, Vol.45(2), pp.196-214.

28) Stechkina, I. B. and Fuchs, N. A. (1966): Studies on fibrous aerosol filters - I. Calculation of diffusional deposition of aerosols in fibrous filters, Annals of Occupational Hygiene, Vol.9, pp.59-64.

29) Lee, K. W. and Liu, B. Y. H. (1982): Theoretical study of aerosol filtration by fibrous filter, Aerosol Science and Technology, Vol.1, pp.147-161.

30) Kirsch, A. A. and Stechkina, I. B. (1978): "The theory of aerosol filtration with fibrous filters. In: Shaw, D.T (Ed.), Fundamentals of Aerosol Science” , Wiley, New York.

31) Podgórski, A. (2009): Estimation of the upper limit of aerosol nanoparticles penetration through inhomogeneous fibrous filters, Journal of Nanoparticle Research, Vol.11, pp.197-207.

\section{Author's short biography}

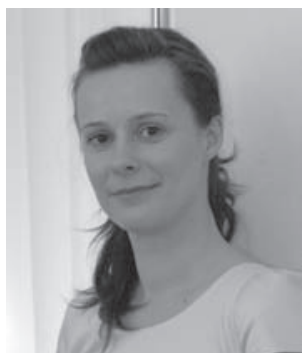

\section{Anna Jackiewicz}

Anna Jackiewicz, PhD, MSc, Assistant Professor at the Faculty of Chemical and Process Engineering Warsaw University of Technology, Poland 2004: MSc - Chemical Engineering; 2010: PhD - Chemical Engineering

Scientific interests: filtration, research of filter effectiveness, optimization of filter structure, techniques for aerosol generation.

Author and co-author of over 40 papers published in periodicals and conference proceedings.

Recipient of the awards: Ignacy Łukasiewicz Grant Fund of PGNiG S.A., Fiat award, the presentation of achievements at the exhibition entitled "Maria SkłodowskaCurie in the Service of Science Yesterday and Today" at the European Parliament in Brussels. 


\section{Author's short biography}

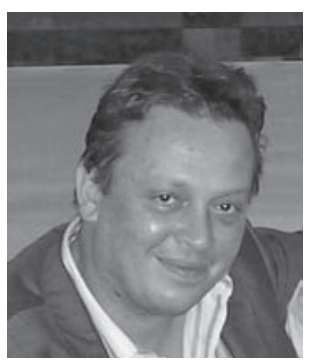

\section{Albert Podgórski}

Albert Podgórski (1963-2010), PhD, DSc, Professor at the Faculty of Chemical and Process Engineering Warsaw University of Technology, Poland.

1988: MSc - Chemical Engineering; 1991: PhD - Chemical Engineering; 2003: DSc - Chemical Engineering; 1995-96 - postdoc, Delft University of Technology (The Netherlands); 1992 - research fellow, Lund University of Technology (Sweden); 1999-2002, 2009 - visiting professor, University of Vienna (Austria).

Scientific interests: interfacial phenomena, dynamics of pulmonary surfactants, particle deposition and clearance in the lung, mechanics of aerosol particles including deformable fibers, aerosol filtration, mathematical modeling.

Author and co-author of over 200 scientific papers, 3 patents, 1 book.

Laureate of the scientific awards: Polish Ministry of Science and Education, Rockefeller Foundation.

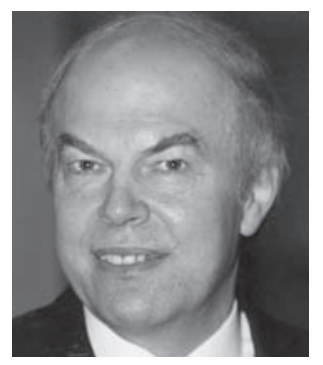

\section{Leon Gradoń}

$\mathrm{PhD}$, DSc, Professor at the Faculty of Chemical and Process Engineering Warsaw University of Technology, Poland.

1969: MSc - Chemical Engineering; 1975: MSc - Mathematics; 1976: PhD - Chemical Engineering; 1978-79 -postdoc, University of Houston (USA); 1985-87 - visiting professor, Buffalo University (USA); 1993-94 - University of Cincinnati (USA); lecturer in European and North American universities.

Scientific interests: filtration, filter production, polymer processing, particle deposition in lungs, controlled drug delivery, functional particle formation.

Author and co-author of over 200 peer-reviewed papers, 60 patents and 3 books.

Recipient of the awards: Smoluchowski, Fulbright, Polish Science Foundation, Japan Society for Promotion of Sciences.

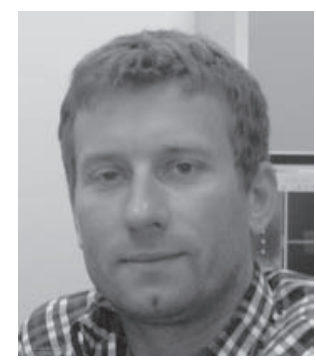

\section{Jakub Michalski}

$\mathrm{PhD}, \mathrm{R} \& \mathrm{D}$ Manager in Materials Engineers Group Ltd; research position at Faculty of Materials Science, Warsaw University of Technology, Poland.

2001: MSc - Materials Science, 2007: PhD - Materials Science; 2004-2005 - EU Marie Curie Fellowship, Josef Stefan Institute, Slovenia; 2002-2004 - Scholarship of Foundation for Polish Science.

Scientific interests: Materials Science, filtration, investment casting of super alloys, composite materials, nano-composites and robotics.

Author and co-author of 16 papers and 1 patent. 